\title{
The diagnostic accuracy of direct agglutination test for serodiagnosis of human visceral leishmaniasis: a systematic review with meta-analysis
}

Mehdi Mohebali, ${ }^{1,2}$, Hossein Keshavarz ${ }^{1,2}$, Sedigheh Shirmohammad ${ }^{2}$, Behnaz Akhoundi ${ }^{1}$, Alireza Borjian ${ }^{1}$, Gholamreza Hassanpour ${ }^{2}$, Setareh Mamishi ${ }^{3}$ and Shima Mahmoudi ${ }^{3^{*}}$

\begin{abstract}
Background: Direct agglutination test (DAT) as a simple, accurate and reliable method, has been widely used for serodiagnosis of visceral leishmaniasis $(\mathrm{VL})$ during the last three decades. The present study is a systematic review and meta-analysis to evaluate the diagnostic accuracy of DAT for serodiagnosis of human VL.

Methods: Electronic databases, including MEDLINE (via PubMed), SCOPUS, Web of Science, SID and Mag Iran (two Persian scientific search engines) were searched from December 2004 to April 2019. We determined the pooled sensitivity and specificity rates of DAT for the diagnosis of human VL, calculated positive and negative likelihood ratios ( $L R+$ and $L R-$ ), and constructed summary receiver operating characteristic (ROC) curves parameters across the eligible studies.
\end{abstract}

Results: Of the 2928 records identified in the mentioned electronic databases and after examining reference lists of articles, 24 articles met inclusion criteria and were enrolled in the systematic review and out of them 20 records qualified for meta-analysis. The pooled sensitivity and specificity rates of DAT was 96\% [95\% Cl, 92-98] and 95\% [Cl95\% 86-99], respectively. The likelihood ratio of a positive test (LR+) was found to be 21 [Cl95\%, 6.6-66.5] and the likelihood ratio of a negative test (LR-) was found to be 0.04 [(Cl95\%, 0.02-0.08]. The combined estimate of the diagnostic odds ratio for DAT was high [467 (C195\%, 114-1912]). We found that the summary receiver operating characteristic curve (SROC) is positioned near the upper left corner of the curve and the area under curve (AUC) was 0.98 (95\% Cl, 0.97 to 0.99$)$.

Conclusion: Referring to our analysis, we determined that DAT can be considered as a valuable tool for the serodiagnosis of human VL with high sensitivity and specificity. As DAT is a simple, accurate and efficient serological test, it can be recommended for serodiagnosis of human $V L$ particularly in endemic areas.

Keywords: Direct agglutination test, Visceral leishmaniasis, Diagnostic accuracy, Human, Systematic review, Metaanalysis

\footnotetext{
* Correspondence: sh-mahmoudi@sina.tums.ac.ir

${ }^{3}$ Pediatric Infectious Diseases Research Center, Tehran University of Medical Sciences, No. 62, Dr. Gharib St., Tehran, Iran

Full list of author information is available at the end of the article
}

C C The Author(s). 2020 Open Access This article is licensed under a Creative Commons Attribution 4.0 International License, which permits use, sharing, adaptation, distribution and reproduction in any medium or format, as long as you give appropriate credit to the original author(s) and the source, provide a link to the Creative Commons licence, and indicate if changes were made. The images or other third party material in this article are included in the article's Creative Commons. licence, unless indicated otherwise in a credit line to the material. If material is not included in the article's Creative Commons licence and your intended use is not permitted by statutory regulation or exceeds the permitted use, you will need to obtain permission directly from the copyright holder. To view a copy of this licence, visit http://creativecommons.org/licenses/by/4.0/. The Creative Commons Public Domain Dedication waiver (http://creativecommons.org/publicdomain/zero/1.0/) applies to the data made available in this article, unless otherwise stated in a credit line to the data. 


\section{Background}

Visceral leishmaniasis (VL) is one of the most important neglected tropical diseases that is caused by Leishmania donovani and L. infantum/chagasi in both humans and canines [1-3]. The morbidity and mortality due to VL are estimated at 200,000 to 400,000 new cases a year and approximately 20,000 to 40,000 deaths occur annually [4]. More than 90\% of all VL cases occur in 6 countries including India, Bangladesh, Brazil, Ethiopia, Sudan, and South Sudan [5].

Since the fatality rate of VL is high and it can reach $100 \%$ when not treated properly, a diagnostic test with both high validity and reproducibility rates is required $[1,6]$.

Early diagnosis and treatment is the first step for elimination of anthroponotic form of VL caused by L.donovani because post kala-azar dermal leishmaniasis (PKDL) cases are potential reservoir hosts in the endemic areas of Indian form of kala-azar, while in Mediterranen form of kala-azar caused by L.infantum/ chagasi, infected canines are potential role as reservoir hosts of the disease $[1,4]$.

Treatment of VL cases should be recommended after confirmation of the disease.

Ambisome ${ }^{\odot}$ in VL is the first line treatment in pregnancy, for patients whose condition is severe and HIV co-infected patients [1]. A follow-up visit 6 months after the end of treatment is recommended to make sure the treatment was successful. The gold standard for diagnosing VL is mainly parasitological examinations including the demonstration of parasites by microscopic examination of splenic or bone-marrow aspiration $[4,6]$.

Sample preparation for parasitological examinations of $\mathrm{VL}$ is highly invasive. In spite of the high specificity of parasitological examinations, the sensitivity of these methods depends on the type and preparation of samples [7]. Moreover, the accuracy and precision of parasitological examinations is also subject to the experience of the laboratory microscopist. Molecular methods including polymerase chain reaction (PCR)-based methods have been developed and assessed for human and canine VL using various target genes and different clinical specimens [8]. The pooled sensitivity and specificity of PCR for detection of infections caused by L.infantum/chagasi on human peripheral blood samples were reported as 93.1 and $95.6 \%$, respectively $[7,8]$. The main limitation of PCR assays is the lack of standardization due to the large numbers of different administrative protocols [7]. In addition, the specificity of the molecular methods varied significantly in different studies in the literature due to the fact that in some studies parasitological methods are not able to identify true-positive patients [7]. Moreover, these methods require specific and expensive equipment and materials and also the performance of molecular methods particularly in remote areas has some limitations.

Several serological tests are used for serodiagnosis of VL, including the indirect fluorescent antibody test (IFAT), the enzyme linked immunosorbent assay (ELIS A), the latex agglutination test (LAT), immunoblotting, the direct agglutination test (DAT) and the rK39 rapid diagnostic test. Among which, DAT and rK39 are simple and do not require sophisticated equipment; thus, they are usually used in field studies as well as in laboratories and seems to be a better screening test particularly in symptomatic VL.

One of the major drawbacks of serological tests is that they are not able to detect relapses among VL cases because anti-Leishmania antibodies remain in the body for a long time after clinical cure $[9,10]$ and many people who live in endemic areas have anti-Leishmania antibody titers due to high exposure to Leishmania parasites. In the previous meta-analysis of the diagnostic performance of the DAT for VL from January 1986 to December 2004, the pooled sensitivity and specificity rates were calculated as 94.8 and $86 \%$, respectively [11]. This study aimed to update the diagnostic accuracy of the DAT for human VL diagnosis from December 2004 to April 2019.

\section{Methods}

\section{Study design}

In this study, all the studies related to diagnostic performance of DAT during 2004-2019 were systematically searched.

Selection of articles was made independently by two reviewers ( $\mathrm{SSH}$ and $\mathrm{AB}$ ) and the possible discrepancies were solved by consensus after discussion.

This systematic review with meta-analysis was conducted as per PRISMA (Preferred Reporting Items for Systematic Reviews and Meta-Analysis) guidelines [12].

\section{Search strategy}

Electronic databases, including MEDLINE (via PubMed), SCOPUS, Web of Science and two Persian scientific search engines "Scientific Information Database" (www. sid.ir) and "Mag Iran" (www.magiran.com) were searched systematically with various combinations of the following scientific keywords: "Visceral leishmaniasis", "Leishmania infantum", "Leishmania donovani" "DAT", "Direct Agglutination Test", "Parasitology", "Microscopy", "Specificity" and "Sensitivity" using "OR" and/or "AND". The references of included articles were additionally screened manually. Abstracts of articles which published in congresses were not explored. 


\section{Case definition}

Patients in VL-endemic countries with pathogonomonic signs and symtomes such as fever $>2$ weeks, hepatosplenomegaly, progress weakness, anaemia or pancytopenia, and lymphadenopathy with confirmation of following testsparticularly in VL endemic areas were considered as VL cases.

\section{Parasitological diagnosis}

(a)

Microscopic findings of bone marrow aspirate, lymph node aspirate, and/or spleen aspirate for detection of amastigote form of Leishmania spp. using direct semiquantified microscopic examination of smears.

(b)

Culture of bone marrow, lymph node, and/or spleen aspirations in Novy-MacNeal-Nicolle (NNN) media. Demonstration of at least one amastigote upon microscopic examination of tissue smears or one promastigote in culture is sufficient for the diagnosis.

\section{Serological tests}

DAT was performed on each sample in standardized conditions, and each test was read by two readers who were blind to other test results.

\section{Study selection and data extraction}

Titles and abstracts of all articles were screened by one reviewer, and eligibility of the screened articles was assessed by two independent investigators using the following criteria.

Inclusion criteria were: a) have a full description of accuracy of DAT as a diagnostic test for the detection of VL in patients. b) Articles were published from December 2004 to April 2019. c) including both DAT test and parasitological examinations (direct demonstration or culture) as the diagnostic method for VL. c) Cases with no previous history of VL were included.

Exclusion criteria were: a) insufficient primary and/ data for calculating of sensitivity and specificity), b) unavailable full text article, and c) written in a language other than English or Persian.

Eligibility of all explored papers was assessed by three reviewers. All possible discrepancies among studies were resolved by discussion and consensus. The extracted data were gathered using a pre-designed form containing all the descriptive variables and test results. The following information was extracted: authors, year and country in which the study was carried out, diagnostic methods applied, reference test used, cut off, characteristics of the participants, quality of the study and sample size.

\section{Assessment of study quality}

We assessed the quality of studies using the Quality Assessment of Studies of Diagnostic Accuracy ApproachQUADAS [13].

\section{Data synthesis and statistical analysis}

All meta-analysis methods were performed using STATA (Release 12. statistical software. College Station, Texas: STATA Corp LP). Sensitivity and specificity were calculated using the available extracted data. To calculate sensitivity and specificity values for the tests, we extracted raw data from primary studies to calculate true positives, false positives, true negatives, and false negatives.

In the cases that the numbers of true positive, false negative, true negative, and false positive observations were not available, we derived the numbers from the marginal totals and the reported sensitivity and specificity.

Meta-analyses were accomplished by using randomeffects inverse-variance weights. Results of the metaanalysis were illustrated by a forest plot diagram, which demonstrated the accuracy estimates and their relevant 95\% confidence interval (CI).

The Cochran's $Q$ and $I^{2}$ statistics were used for assessment of the between-study inconsistency and heterogeneity, respectively. The $I^{2}$ values of 25,50 , and $75 \%$ were representatives of low, moderate and high heterogeneity, respectively. Publication bias was evaluated through the Deeks' funnel plots [14].

\section{Results}

From the literature searches, we identified 2928 primary citations from electronic databases and through articles' reference lists. Study selection flow is shown in Fig. 1.

Among all records, 24 articles met inclusion criteria and included into the systematic review. The characteristics of the included studies are summarized in Table 1.

The reference method for diagnosis of VL in all studies was a positive result on microscopic examination and/or culture of lymph node, bone marrow, or spleen aspirates $[25,28,34]$.

Some studies performed DAT using both freeze-dried (FD) and aqueous (AQ) antigen [18, 37]. No significant difference in the sensitivity of the DAT (FD and AQ) was found. In one study [37], relatively higher sensitivity (99\%) was recorded for the LQ-DAT than for the FDDAT (96\%) that might be due to the use of the endemic autochthonous Leishmania donovani isolate as the antigen.

In overall, studies had a wide geographical distribution and were carried out in different countries including Brazil $[16,35,36]$, Ethiopia [28, 30], India [15, 17, 18, 24, 26, 32], Iraq [31], Iran [20, 23, 27], Saudi Arabia [34], 


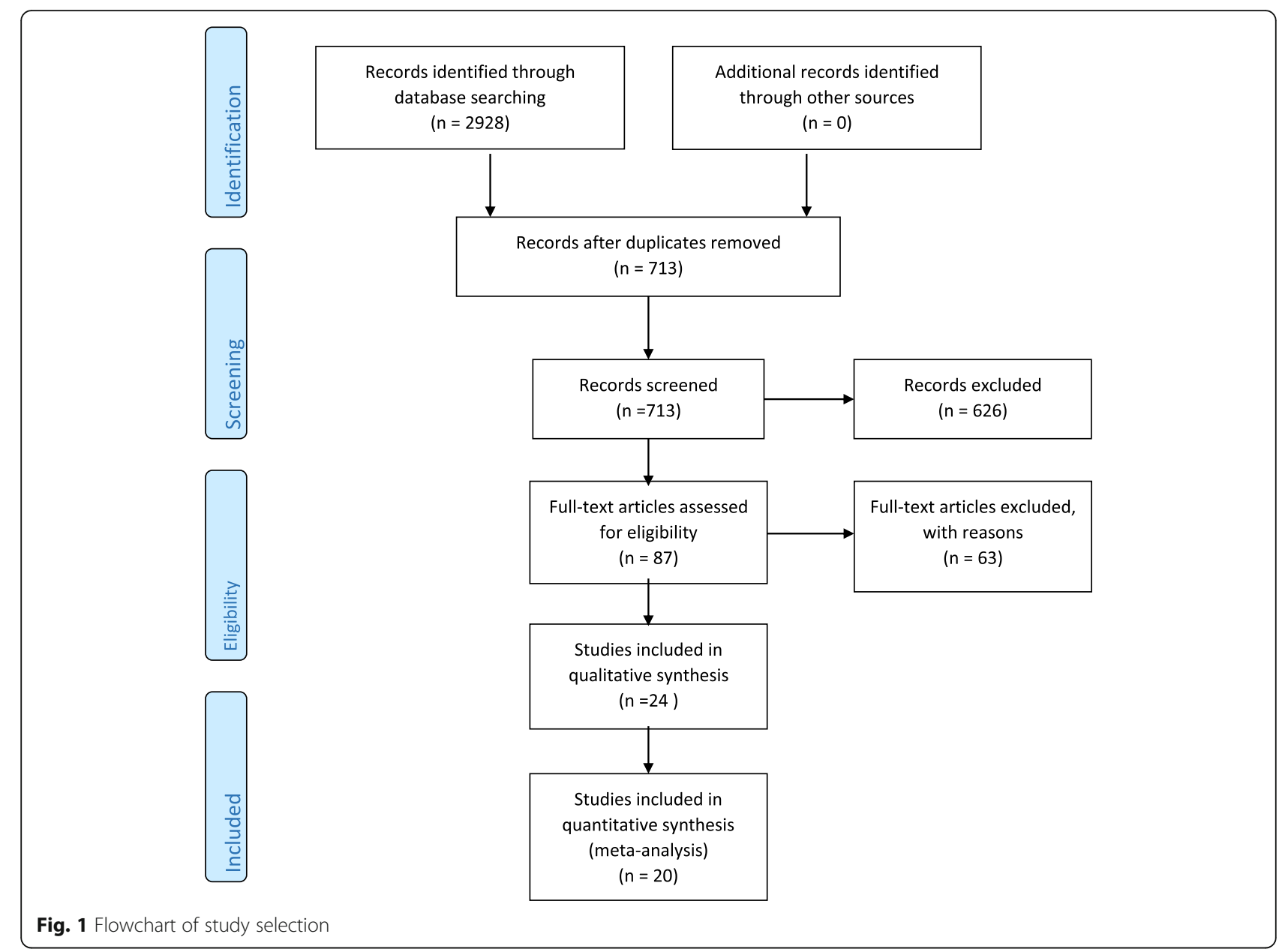

Sudan $[19,21,29,33,37]$, Turkey [25] and Venezuela [22].

The study quality analysis as assessed by QUADAS tool showed that 22 out of 24 studies (92\%) met more than seven criteria (Table 1).

Among 24 studies, 20 records were qualified for metaanalysis. All studies except 2 reports (one cohort [32] and one case-control [34] were cross sectional studies evaluating the diagnostic accuracy of DAT and metaanalysis was performed on all cross sectional studies evaluating the diagnostic accuracy of DAT. Three studies were excluded because there was zero cell and sensitivity and/or specificity cannot be calculated. Cut off dilution for a positive test on DAT reported to vary between $1: 800$ to $1: 3200$ (Table 2).

Figures 1 shows the results of individual and combined sensitivity and specificity estimates of the DAT test for diagnosis of VL. The pooled sensitivity of the included studies evaluating the DAT was 96\% [CI95\% 92-98] and the pooled specificity was 95\% [CI95\% 86-99] (Fig. 2). The likelihood ratio of a positive test (LR+) was found to be 21 [CI95\% 6.6-66.5] and the likelihood ratio of a negative test (LR-) was found to be 0.04 [CI95\% - 0.02-
0.08] (Fig. 3). Moreover, the combined estimate of the diagnostic odds ratio for DAT was high (467 [1141912]).

Among studies which considered DAT cut off value of $1: 800$, the pooled sensitivity and specificity was 95\% [CI95\% 66-100] and 99\% [CI95\% 93-100], respectively. With considering this cut off value, the $\mathrm{LR}+$ was found to be 107 [C195\% 12.7-901] that was more higher than LR+ obtained from analysis of studies with cut off value of 1:1600 and 1:3200. Although the pooled sensitivity of DAT with cut off value of 1 : 1600 and 1:3200 was remained stable (97 and 96\%, respectively), the pooled specificity was declined to $87 \%$ [C195\%83-90] with cut off value of $1: 1600$ and 93\% [CI95\% 73-99] with cut off value of 1:3200, respectively.

The pooled sensitivity and specificity of DAT for serodiagnosis of $L$. donovani was $96 \%$ [CI95\% 92-98] and 98\% [CI95\% 93-99], respectively; while it was 92\% [CI95\% 83-96] and 98\% [CI95\% 15-100] for serodiagnosis of $L$. infantum, respectively (Table 2).

We found that the summary receiver operating characteristic curve (SROC) is positioned near the upper left 


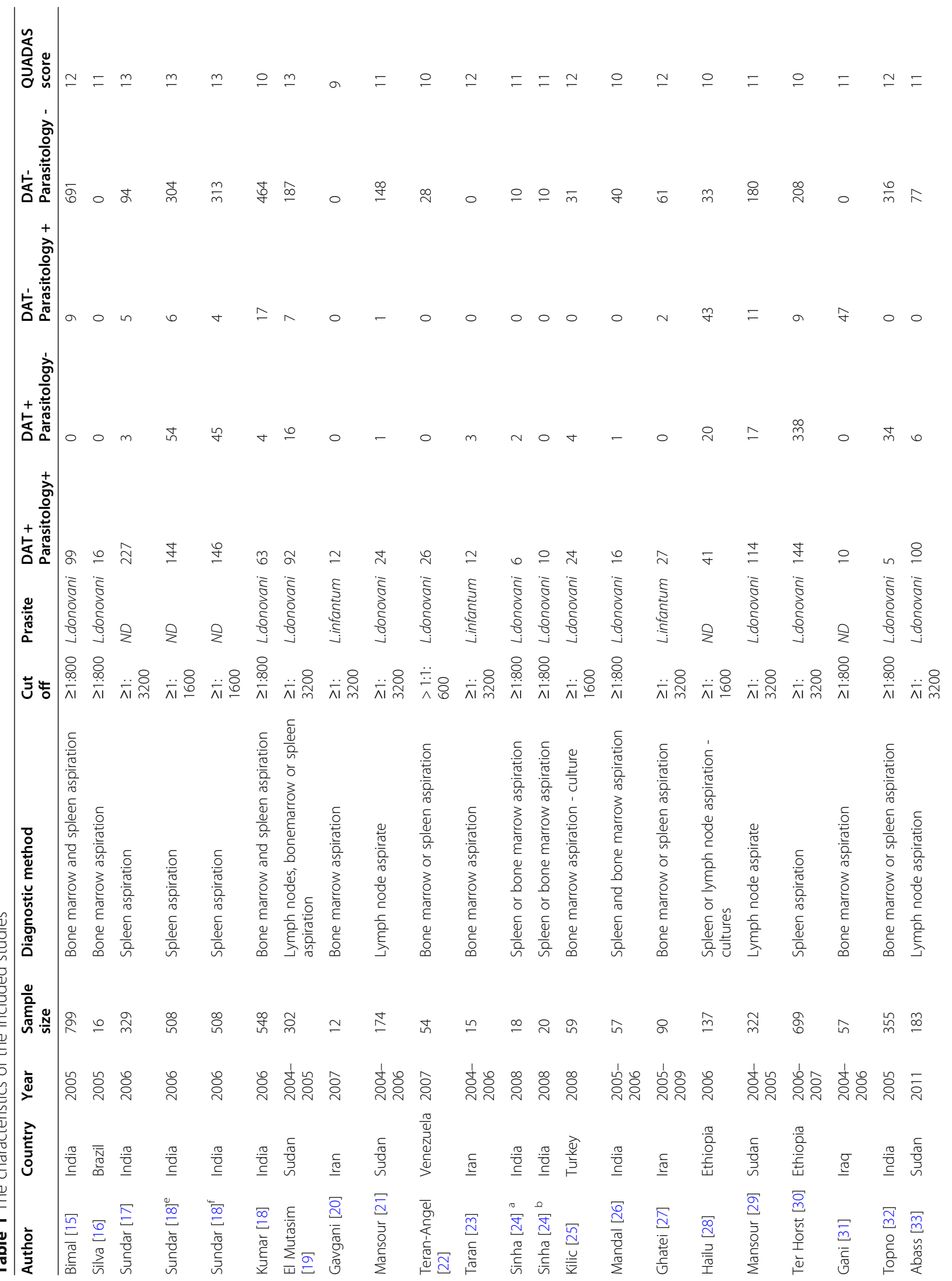




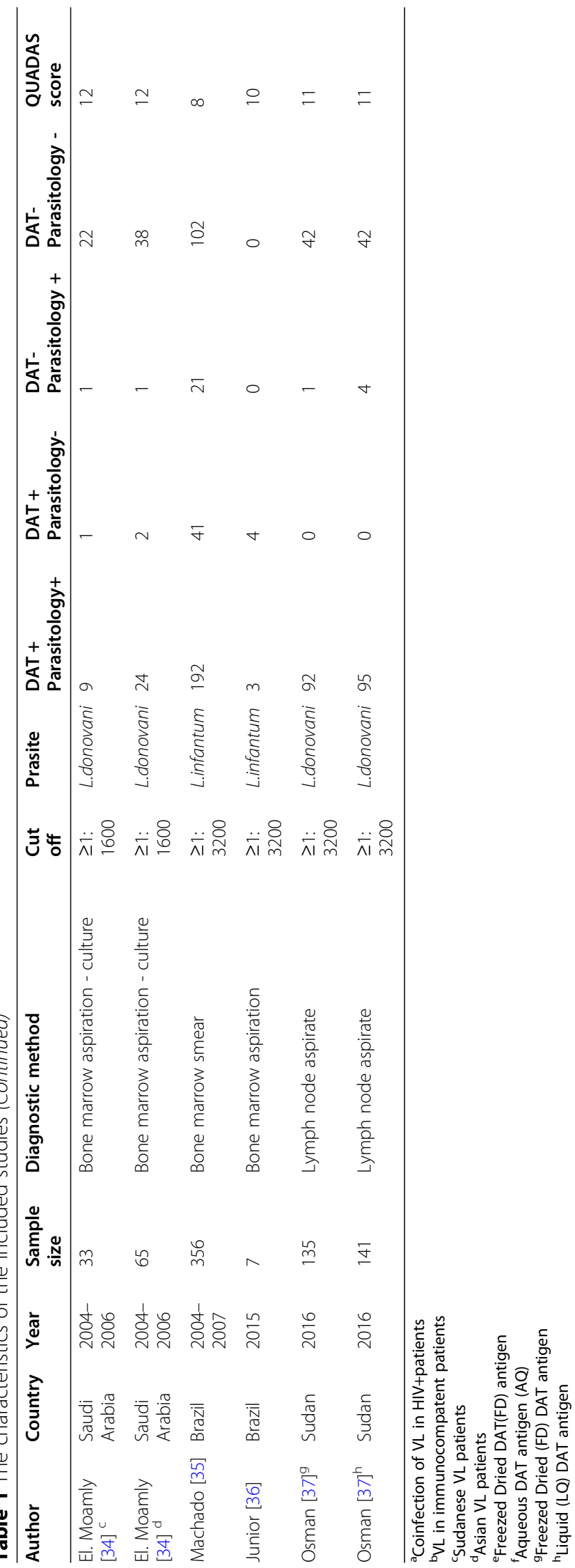


Table 2 Diagnostic accuracy of DAT for diagnosis of VL based on different cut off values and Leishmania species

\begin{tabular}{|c|c|c|c|c|c|c|}
\hline \multirow[t]{2}{*}{ Parameter } & \multicolumn{2}{|c|}{ Leishmania species } & \multicolumn{4}{|l|}{ Cut off } \\
\hline & L.donovani & L.infantum & All & $1: 800$ & 1:1600 & 1:3200 \\
\hline Sensitivity & 96 [92-98] & 92 [83-96] & 96 [92-98] & 95 [66-100] & 97 [94-99] & 96 [93-97] \\
\hline Specificity & 98 [93-99] & 98 [15-100] & 95 [86-99] & 99 [93-100] & 87 [83-90] & 93 [73-99] \\
\hline Positive Liklihood Ratio & $48.6[13.6-174]$ & $40[0.2-8793]$ & $21[6.6-66.5]$ & 107 [12.7-901] & $7.5[5.6-10]$ & $14.5[3.1-66.6]$ \\
\hline Negative Liklihood Ratio & $0.04[0.02-0.08]$ & $0.09[0.04-0.18]$ & $0.04[0.02-0.08]$ & $0.05[0.01-0.44]$ & $0.03[0.01-0.07]$ & $0.05[0.03-0.08$ \\
\hline Diagnostic Odds Ratio & 1228 [280-5393] & $467[1-147,442]$ & 467 [114-1912] & $2206[155-31,482]$ & 236 [83-674] & 309 [56-1721] \\
\hline
\end{tabular}

corner of the curve and the area under curve (AUC) was 0.98 (95\% CI, 0.97 to 0.99) (Fig. 4).

The Deeks' funnel plots for publication bias also showed no asymmetry (Fig. 5). The evaluation of publication bias showed no potential for publication bias $(p=$ $0.69)$.

\section{Discussion}

The first line regimen for primary VL treatment is pentavalent antimonial compounds such as meglumine antimoniate (Glucantime ${ }^{\circ}$ ) and Sodium stibogluconate(Pentostam $^{\circ}$ ) which can be used as a monotherapy (administered as intramuscular injections of $20 \mathrm{mg} / \mathrm{kg} /$ day for 28-30 days) or in combination with cryotherapy or other drugs such as paramomycin [1-3]. Other drugs such as amphotericin B deoxycholate, Liposomal amphotericin B (Ambisome ${ }^{\circ}$ ) and miltefosine (administrated orally) may be used for the treatment of VL particularly in patients with clinical or laboratory resistance to pentavalent drugs as well as contraindications caused by these drugs [1].

Until 1990, VL diagnosis was in need of parasitological confirmation including microscopy or culture of the spleen, bone-marrow, lymph nodes and sometimes

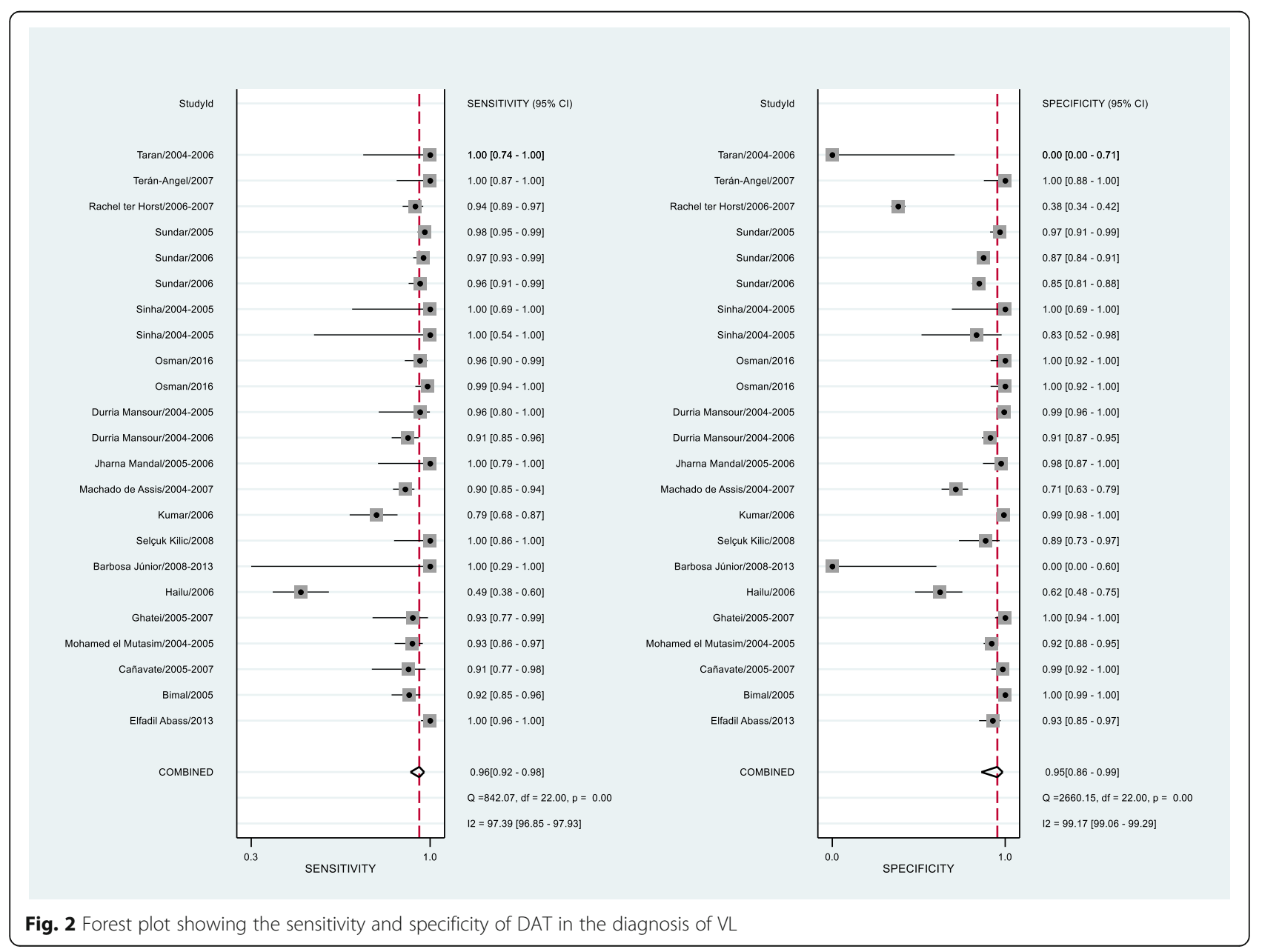




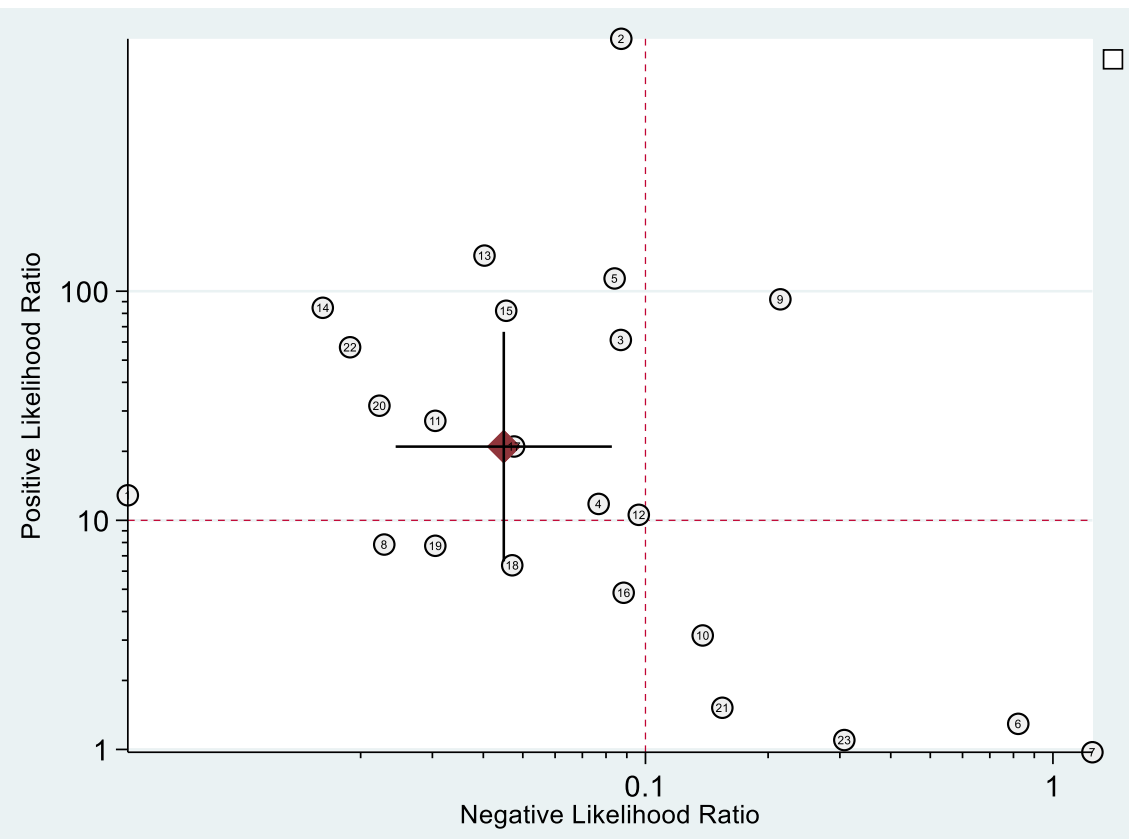

Fig. 3 Positive and negative likelihood ratio of the included studies

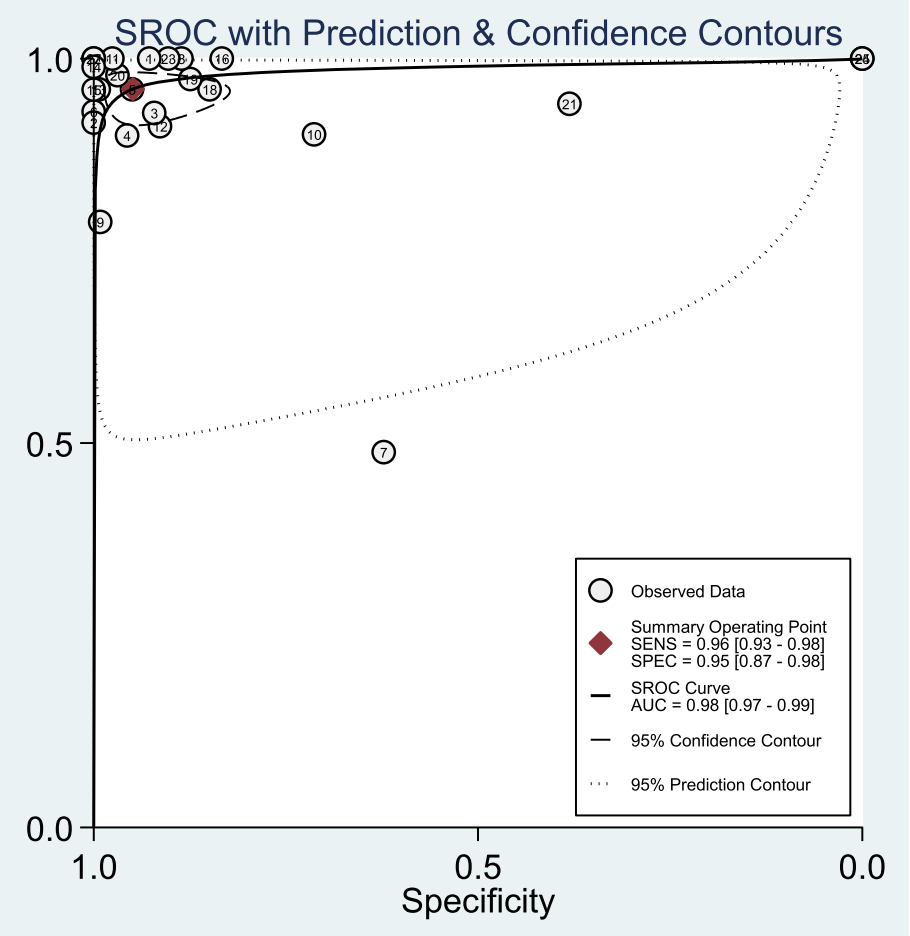

Fig. 4 Summary receiver operating characteristic (SROC) curve for assessment of the diagnostic accuracy of DAT for the diagnosis of VL 


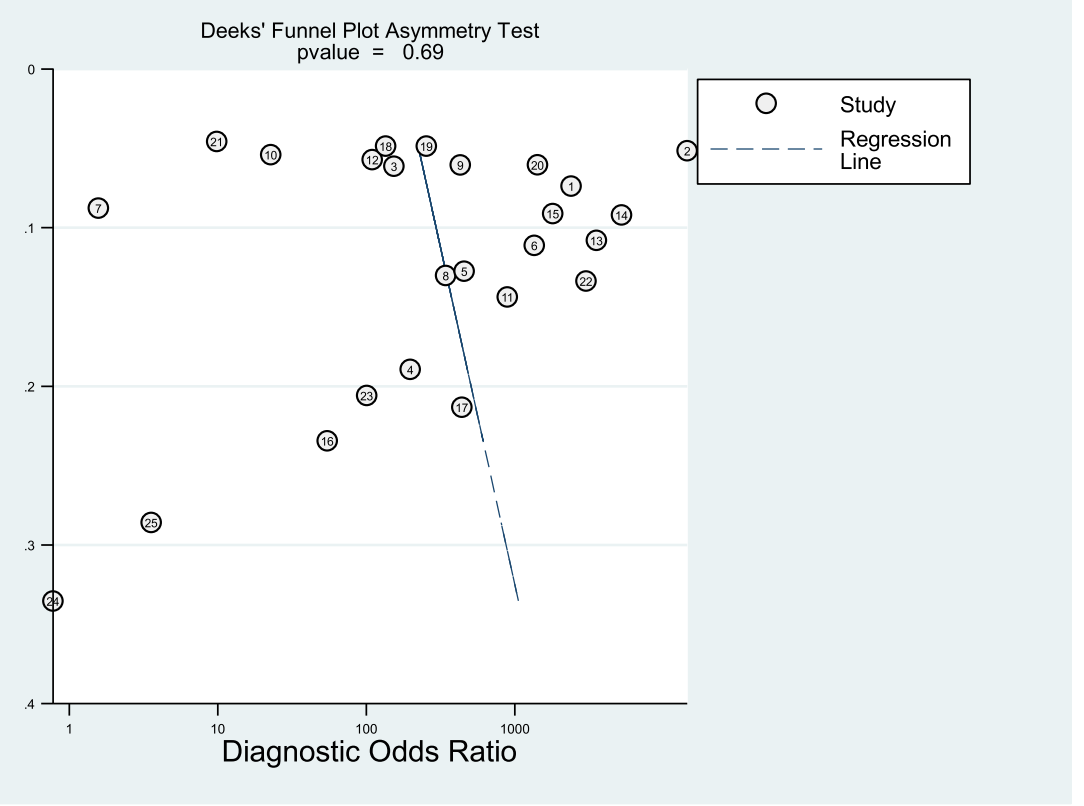

Fig. 5 Deeks' funnel plots for publication bias

peripheral blood specimens. The invasiveness and sometimes fatal complications particularly associated with splenic aspiration brought about the development of simple and accurate serological tests such as DAT [38]. Although the IFAT and ELISA are two important serological methods for diagnosis of human VL they require specific materials and equipment $[1,33]$. The rK39 dipstick is simpler than DAT for screening of human VL particularly in symptomatic cases. The rK39-based tests are easy to perform, quick, cheap and give reproducible results and can therefore be used for early diagnosis of $\mathrm{VL}$ at both peripheral and central levels of public health centers Studies comparing the rK39 strip test and DAT found a similar sensitivity (94\%) and specificity (89\%); however, the DAT showed a slightly higher specificity [39].

On the other hand, rK39 strip tests have certain limitations because anti-Leishmania antibodies can persist for months in patients even after recovery, moreover, it has low specificity in sub-clinical and asymptomatic forms of L.donovani or L.inantum/chagasi infections, particularly among the Sudanese population [33, 37]. A recent introduced assay based on the detection of antibodies to the rk28 fusion protein showed a very promising sensitivity and specificity (96 and 98\%, respectively) of ELISA to detect anti-Leishmania antibodies in sera among VL patients. In addition, The rK26, A2-ELISA and rKE16 dipstick recombinant antigens from amastigote forms of L.infantum or L.donovani showed desirable results [40-42]. Among the available serological tests for the diagnosis of VL, DAT is a simple, highly specific and sensitive, reliable and cost-effective test that can be used in field as well as laboratory studies [4347]. DAT as a semi-quantitative serological test has been used for the serodiagnosis and seroepidemiological studies of VL in both humans and animal reservoir hosts during the last 4 decades. According to our knowledge, this is the second systematic review and meta-analysis about the diagnostic accuracy of DAT for diagnosis of VL. Chappuis et al. included 30 relevant studies that evaluated DAT from January 1986 to December 2004 [11] and reported the pooled sensitivities and specificities of 94.8 and $86 \%$, respectively. In the present study, 24 eligible studies from April 2004 to December 2019 were evaluated using DAT for the diagnosis of VL in immunocompetent patients by the systematic review. Our meta-analysis showed DAT is still a validated serodiagnostic test with high pooled sensitivity of 95\% [CI95\%, 66-100], 97\% [CI95\%,94-99] and 96\% [CI95\%, 93-97], respectively while 1:800, 1:1600, and1:3200 cut-off titer were considered. Higher pooled specificity $99 \%$ (95\% CI, 93-100) was found at a 1:800 cut-off titer.

Specific Leishmania antibodies at a titer of 1:800 showed VL infection [33] whereas Leishmania antibodies at a titer of 1:3200 with pathogonomonic clinical signs such as hepatosplenomegaly, dromedary fever, anemia and progress weakness reflected active VL [43].

The high diagnostic accuracy of DAT using different samples including serum, plasma or even urine samples has been reported [11, 48]. 
According to the of the previous meta-analysis comparing the diagnostic accuracy of DAT and rK39 strip test, DAT showed $1 \%$ more sensitivity and $2 \%$ more specificity than rK39 strip test.

Although we did not have sufficient information regarding human immunodeficiency viruses status of the cases, it has been reported that DAT has an acceptable sensitivity in the diagnosis of VL in HIV-positive patients [49]. Although sensitivity of DAT in the majority of studies was more than $90 \%$ some heterogeneity in the sensitivity of the tests might be related to the geographical location of the study, differences in antibody concentrations, and immune or nutritional status of the patient [50]. In the current study, it was not possible to evaluate the test performance according to these factors.

Although early diagnosis and appropriate treatment is crucial for controling of the anthroponotic form of VL (i.e. Indian and African forms of VL), control of zoonotic VL is highly difficult and the current control strategies for zoonotic VL rely on animal reservoir hosts and phlebotomine vectors [1], the use of insecticide-impregnated materials to prevent insect bites, and active case detection with appropriate treatment to decrease the mortally rate of VL [45].

VL is highly fatal in the absence of appropriate antiLeishmania drugs. Although pentavalent antimonial compounds are introduced as the drugs of choice for the treatment of $\mathrm{VL}$, intramuscular or painful intravenous injections with high toxicity and high price consider. However, anti-leishmanial drugs are usually expensive and have significant toxicity. Since the clinical manifestations of VL have low specificity and are not completely pathognomonic, performing of confirmatory tests to recognize the VL patients needing treatment are highly required. These tests must not only be sensitive, but they also need to be specific because the current anti-Leishmania drugs prescribed to treat VL are highly toxic [7].

The most important limitation for the evaluation of serological tests is the absence of an appropriate gold standard test. Confirmation of VL depends on the finding of Leishman bodies of Leishmania sp. in samples prepared from bone marrow or spleen, lymph nodes and liver. This procedure is highly invasive, and it should be performed only in suspected cases of VL. Furthermore, the sensitivity of this method is diverse [47].

In some VL-endemic areas, DAT is used regularly for the diagnosis and sero-epidemiological studies of VL because it is simple due to its high sensitivity [33, 44].

The performance of DAT is neither Leishmania species-specific nor region dependent $[6,43]$.

Major limitations of DAT are its long incubation period to report, batch to batch variability of the antigen and cross-reactivity with Trypanosoma cruzi infection $[43,44]$. DAT titers decline over time below the cut off
(1:800), while itstill remain positive for a relatively long time (up to 5 years in more than $50 \%$ of VL cases) after the cure. Therefore, performing of this test for treatment follow up or for the diagnosis of disease relapse is not recommended [49]. To overcome the problem of long incubation time, a fast agglutination screening test (FAST) has been introduced, which uses only one serum dilution and requires only three hours of incubation [51]. FAST for the detection of L. infantum infection was compared with the conventional DAT in Iran indicating a sensitivity of $95.4 \%$ and specificity of $88.5 \%$ for fast DAT in comparison with conventional DAT [51].

Our study has some limitations. First, we compared pooled estimates between different published studies. So, the possibility of confounding should not be ignored. Second, the study used the data provided by published literature, and some data including sex and age for the included patients was unavailable and made subgroup analysis difficult.

\section{Conclusion}

Based on our analysis, the use of DAT is the best choice for early detection of acute and chronic clinical forms of $\mathrm{VL}$ as well as the asymptomatic form of the infections in immunocompenent individuals. Further research on DAT antigen standardization in the laboratories located in endemic areas of $\mathrm{VL}$ is recommended.

\section{Abbreviations}

DAT: Direct agglutination test; VL: Visceral leishmaniasis; FAST: Fast agglutination screening test; PCR: Polymerase chain reaction; IFAT: Indirect fluorescent antibody test; ELISA: Enzyme linked immunosorbent assay; FD: Freeze-dried; AQ: Aqueous antigen; SROC: Summary receiver operating characteristic curve; AUC: Area under curve

\section{Acknowledgements}

Not Applicable.

\section{Authors' contributions}

MM: involved in designing, interpretations and writing of the manuscript. HK, SM1 and GH: participated in interpretation of the DAT results. SS, BA and AB: involved in gathering and grouping the articles regarding DAT for visceral leishmaniasis and drafted the manuscript. SM2: involved in data analysis and writing of the manuscript. All authors read and approved the final manuscript.

\section{Funding}

This study was approved and financially supported by Tehran University of Medical.

Sciences (TUMS) (Project No: 97-01-160-37931). The role of the funding was for body design of the study, collection, analysis, interpretation of data and writing the manuscript.

\section{Availability of data and materials}

The datasets used and/or analysed during the current study available from the corresponding author on reasonable request.

Ethics approval and consent to participate Not applicable.

Consent for publication

Not applicable. 


\section{Competing interests}

The authors declare that they have no competing interests.

\section{Author details}

'Department of Medical Parasitology and Mycology, Tehran University of Medical Sciences, School of Public Health, Tehran, Iran. ${ }^{2}$ Center for Research of Endemic Parasites of Iran (CREPI), Tehran University of Medical Sciences, Tehran, Iran. ${ }^{3}$ Pediatric Infectious Diseases Research Center, Tehran University of Medical Sciences, No. 62, Dr. Gharib St., Tehran, Iran.

Received: 23 March 2020 Accepted: 30 October 2020 Published online: 11 December 2020

\section{References}

1. Organization WH. Report of a meeting of the WHO expert committee on the control of Leishmaniases, Geneva, Switzerland, 22-26 March 2010. WHO Technical Report Series 2010 (949).

2. Naeem AT, Mahmoudi S, Saboui F, Hajjaran H, Pourakbari B, Mohebali M, et al. Clinical features and laboratory findings of visceral leishmaniasis in children referred to children medical center hospital, Tehran, Iran during 2004-2011. Iran J Parasitol. 2014;9(1):1-5.

3. Abdolsalehi M, Pourakbari B, Mahmoudi S, Moradzadeh M, Keshavarz H, Mamishi S. Clinical and epidemiologic features of visceral Leishmaniasis in children: a 6-year study from an Iranian referral hospital. Infect Disord Drug Targets. 2020;20(4):461-6.

4. Alvar J, Vélez ID, Bern C, Herrero M, Desjeux P, Cano J, et al. Leishmaniasis worldwide and global estimates of its incidence. PLoS One. 2012;7(5): e35671.

5. World Health Organization. WHO to implement online epidemiological surveillance for leishmaniasis, 2016. 2016.

6. Sarkari B, Rezaei Z, Mohebali M. Immunodiagnosis of visceral leishmaniasis: current status and challenges: a review article. Iran J Parasitol. 2018;13(3): 331-41.

7. Chappuis F, Sundar S, Hailu A, Ghalib H, Rijal S, Peeling RW, et al. Visceral leishmaniasis: what are the needs for diagnosis, treatment and control? Nat Rev Microbiol. 2007;5(11supp):S7.

8. De Ruiter C, Van der Veer C, Leeflang M, Deborggraeve S, Lucas C, Adams E. Molecular tools for diagnosis of visceral leishmaniasis: systematic review and meta-analysis of diagnostic test accuracy. J Clin Microbiol. 2014;52(9):314755.

9. Hailu A. Pre-and post-treatment antibody levels in visceral leishmaniasis. Trans R Soc Trop Med Hyg. 1990;84(5):673-5.

10. Silva LA, Romero HD, Prata A, Costa RT, Nascimento E, CARVALHO SFG, et al. Immunologic tests in patients after clinical cure of visceral leishmaniasis. Am J Trop Med Hyg. 2006;75(4):739-43.

11. Chappuis F, Rijal S, Soto A, Menten J, Boelaert M. A meta-analysis of the diagnostic performance of the direct agglutination test and rK39 dipstick for visceral leishmaniasis. BMJ. 2006;333(7571):723.

12. Moher D, Liberati A, Tetzlaff J, Altman DG. Preferred reporting items for systematic reviews and meta-analyses: the PRISMA statement. Ann Intern Med. 2009;151(4):264-9.

13. Whiting P, Rutjes AW, Reitsma JB, Bossuyt PM, Kleijnen J. The development of QUADAS: a tool for the quality assessment of studies of diagnostic accuracy included in systematic reviews. BMC Med Res Methodol. 2003;3(1):25.

14. Deeks JJ, Macaskill P, Irwig L. The performance of tests of publication bias and other sample size effects in systematic reviews of diagnostic test accuracy was assessed. J Clin Epidemiol. 2005;58(9):882-93.

15. Bimal S, Das VN, Sinha PK, Gupta AK, Verma N, Ranjan A, et al. Usefulness of the direct agglutination test in the early detection of subclinical Leishmania donovani infection: a community-based study. Ann Trop Med Parasitol. 2005;99(8):743-9.

16. Silva ES, Schoone GJ, Gontijo CM, Brazil RP, Pacheco RS, Schallig HD. Application of direct agglutination test (DAT) and FAST agglutination screening test (FAST) for sero-diagnosis of visceral leishmaniasis in endemic area of Minas Gerais, Brazil. Kinetoplastid Biol Dis. 2005;4:4.

17. Sundar S. Serological diagnosis of Indian visceral leishmaniasis - Direct agglutination test versus RK39 strip test and comparison of two strip formats. Am J Trop Med Hyg. 2006 ;100(6):533-7.

18. Kumar R, Pai K, Kumar P, Pandey HP, Sundar S. Sero-epidemiological study of kala-azar in a village of Varanasi district, India. Tropical Med Int Health. 2006;11(1):41-8.
19. el Mutasim M, Mansour D, Abass EM, Hassan WM, el Harith A. Evaluation of a glycerol-preserved antigen in the direct agglutination test for diagnosis of visceral leishmaniasis at rural level in eastern Sudan. J Med Microbiol. 2006; 55(Pt 10):1343-7.

20. Gavgani ASM, Ghazanchaei A, Karimi P, Mohit H, Davies CR. Practical approach for typing strains of Leishmania infantum by enzyme polymorphism: a cross sectional study in northwest of Iran. Pak J Biol Sci. 2007;10(24):4505-9.

21. Mansour D, Abass EM, El Mutasim M, Mahamoud A, El Harith A. Use of a newly developed beta-mercaptoethanol enzyme-linked immunosorbent assay to diagnose visceral leishmaniasis in patients in eastern Sudan. Clin Vaccine Immunol. 2007;14(12):1592-5.

22. Teran-Angel G, Schallig H, Zerpa O, Rodriguez V, Ulrich M, Cabrera M. The direct agglutination test as an alternative method for the diagnosis of canine and human visceral leishmaniasis. Biomedica. 2007;27(3):447-53.

23. M Taran, M Mohebali, MH Modaresi, S Mamishi, M Mojarad, M Mahmoudi. Preparation of a K39sub Recombinant Antigen for the Detection of Leishmania infantum Antibodies in Human: a Comparative Study with an Immunochromatographic Test and Direct Agglutination. Iranian J Parasitol: Vol.2, No.2, 2007, pp. 25-33.

24. Sinha PK, Bimal S, Pandey K, Singh SK, Ranjan A, Kumar N, et al. A community-based, comparative evaluation of direct agglutination and rK39 strip tests in the early detection of subclinical Leishmania donovani infection. Ann Trop Med Parasitol. 2008;102(2):119-25.

25. Kiliç S, Taylan Özkan A, Babür C, Tanir G, Schallig HDFH. Evaluation of serological tests for the diagnosis of visceral leishmaniasis. Turkish J Med Sci. 2008;38(1):13-9.

26. Mandal J, Khurana S, Dubey ML, Bhatia P, Varma N, Malla N. Evaluation of direct agglutination test, rk39 test, and ELISA for the diagnosis of visceral leishmaniasis. Am J Trop Med Hyg. 2008;79(1):76-8.

27. Ghatei MA, Hatam G, Hossini SMH, Sarkari B. Performance of latex agglutination test (KAtex) in diagnosis of visceral leishmaniasis in Iran. Iran J Immunol. 2009;6(4):202-7.

28. Hailu A, Gramiccia M, Kager PA. Visceral leishmaniasis in aba-Roba, SouthWestern Ethiopia: prevalence and incidence of active and subclinical infections. Ann Trop Med Parasitol. 2009;103(8):659-70.

29. Mansour D, Abass EM, Mahamoud A, Harith AE. Qualitative and semiquantitative comparison of an rk39 strip test and direct agglutination test for detection of anti-Leishmania Donovani antibodies in the Sudan. Iran J Immunol. 2009;6(4):208-15.

30. Ter Horst R, Tefera T, Assefa G, Ebrahim AZ, Davidson RN, Ritmeijer K. Field evaluation of rK39 test and direct agglutination test for diagnosis of visceral leishmaniasis in a population with high prevalence of human immunodeficiency virus in Ethiopia. Am J Trop Med Hyg. 2009;80(6):929-34.

31. Gani ZH, Hassan MK, Jassim AM. Sero-epidemiological study of visceral leishmaniasis in Basrah, southern Iraq. J Pak Med Assoc. 2010;60(6):464-9.

32. Topno RK, Das VN, Ranjan A, Pandey K, Singh D, Kumar N, et al. Asymptomatic infection with visceral leishmaniasis in a disease-endemic area in Bihar, India. Am J Trop Med Hyg. 2010;83(3):502-6.

33. Abass E, Mahamoud A, Mansour D, Mohebali M, Harith AE. Validation of a $\beta$ ME ELISA for detection of anti Leishmania donovani antibodies in eastern Sudan. Iran J Immunol. 2011;8(3):150-8.

34. El-Moamly A, El-Sweify M, Hafeez M. Performance of rK39 immunochromatography and freeze-dried direct agglutination tests in the diagnosis of imported visceral leishmaniasis. Parasitol Res. 2012;110(1):349-54.

35. Machado de Assis TS, Rabello A, Werneck GL. Latent class analysis of diagnostic tests for visceral leishmaniasis in Brazil. Tropical Med Int Health. 2012;17(10):1202-7.

36. Júnior WLB, De Araújo PSR, De Andrade LD, Dos Santos AMA, Da Silva MAL, Dantas-Torres F, et al. Rapid tests and the diagnosis of visceral leishmaniasis and human immunodeficiency virus/acquired immunodeficiency syndrome coinfection. Am J Trop Med Hyg. 2015;93(5):967-9.

37. Osman HA, Mahamoud A, Abass EM, Madi RR, Semiao-Santos SJ, El Harith A. Local production of a liquid direct agglutination test as a sustainable measure for control of visceral Leishmaniasis in Sudan. Am J Trop Med Hyg. 2016;94(5):982-6.

38. Organization WH, UNICEF. Visceral leishmaniasis rapid diagnostic test performance. 2011.

39. Maia Z, Lírio M, Mistro S, Mendes CMC, Mehta SR, Badaro R. Comparative study of rK39 Leishmania antigen for serodiagnosis of visceral leishmaniasis: systematic review with meta-analysis. PLoS Negl Trop Dis. 2012;6(1):e1484.

40. Pattabhi S, Whittle J, Mohamath R, El-Safi S, Moulton GG, Guderian JA, et al. Design, development and evaluation of rK28-based point-of-care tests for 
improving rapid diagnosis of visceral leishmaniasis. PLoS Negl Trop Dis. 2010;4(9):e822.

41. Hosseini Farash BR, Mohebali M, Kazemi B, Hajjaran H, Akhoundi B, Raoofian $R$, et al. Cloning of $K 26$ hydrophilic antigen from Iranian strain of Leishmania infantum. Iran J Public Health. 2017;46(10):1359-65.

42. Farahmand M, Khalaj V, Mohebali M, Khalili G, Naderi S, Ghaffarinejad P, et al. Comparison of recombinant A2-ELISA with rKE16 dipstick and direct agglutination tests for diagnosis of visceral leishmaniasis in dogs in northwestern Iran. Rev Soc Bras Med Trop. 2015;48(2):188-93.

43. Mohebali M, EDRISIAN GH, Nadim A, Hajaran H, Akhoundi B, HOUSHMAND $\mathrm{B}$, et al. Application of direct agglutination test (DAT) for the diagnosis and seroepidemiological studies of visceral leishmaniasis in Iran. Iran J Parasitol. 2006:1(1):15-25.

44. Harith A, Kolk A, Kager P, Leeuwenburg J, Muigai R, Kiugu S, et al. A simple and economical direct agglutination test for serodiagnosis and seroepidemiological studies of visceral leishmaniasis. Trans R Soc Trop Med Hyg. 1986:80(4):583-6.

45. Mohebali M, Edrissian GH, Shirzadi MR, Hosseingholizadeh G, Pashaei MH, Ganji A, et al. Integrated visceral leishmaniasis surveillance system in primary care for children in Meshkin-Shahr district, north-western Islamic Republic of Iran. East Mediterr Health J. 2010;16(10):1050-4.

46. Mohebali M, Edrissian GH, Shirzadi MR, Akhoundi B, Hajiaran H, Zarei Z, et al. An observational study on the current distribution of visceral leishmaniasis in different geographical zones of Iran and implication to health policy. Travel Med Infect Dis. 2011;9(2):67-74.

47. Mohebali M. Visceral leishmaniasis in Iran: review of the epidemiological and clinical features. Iran J Parasitol. 2013;8(3):348.

48. Islam MZ, Itoh M, Mirza R, Ahmed I, Ekram AS, Sarder AH, et al. Direct agglutination test with urine samples for the diagnosis of visceral leishmaniasis. Am J Trop Med Hyg. 2004;70(1):78-82.

49. Bangert M, Flores-Chávez MD, Llanes-Acevedo IP, Arcones C, Chicharro C, García E, et al. Validation of rK39 immunochromatographic test and direct agglutination test for the diagnosis of Mediterranean visceral leishmaniasis in Spain. PLoS Negl Trop Dis. 2018;12(3):e0006277.

50. Cota GF, de Sousa MR, Demarqui FN, Rabello A. The diagnostic accuracy of serologic and molecular methods for detecting visceral leishmaniasis in HIV infected patients: meta-analysis. PLoS Negl Trop Dis. 2012;6(5):e1665.

51. Akhoundi B, Mohebali M, Babakhan L, Edrissian G-H, Eslami M-B, Keshavarz $\mathrm{H}$, et al. Rapid detection of human Leishmania infantum infection: a comparative field study using the fast agglutination screening test and the direct agglutination test. Travel Med Infect Dis. 2010;8(5):305-10.

\section{Publisher's Note}

Springer Nature remains neutral with regard to jurisdictional claims in published maps and institutional affiliations.

Ready to submit your research? Choose BMC and benefit from:

- fast, convenient online submission

- thorough peer review by experienced researchers in your field

- rapid publication on acceptance

- support for research data, including large and complex data types

- gold Open Access which fosters wider collaboration and increased citations

- maximum visibility for your research: over $100 \mathrm{M}$ website views per year

At $\mathrm{BMC}$, research is always in progress.

Learn more biomedcentral.com/submissions 